\title{
Conductivity of Doped Two-Leg Ladders
}

\author{
Eugene H. Kim \\ Department of Physics, University of California, Santa Barbara, California 93106-9530 \\ and \\ $\dagger$ Department of Physics and Astronomy, McMaster University, Hamilton, Ontario, Canada L8S-4M1
}

\begin{abstract}
Recently, conductivity measurements were performed on the hole-doped two-leg ladder material $\mathrm{Sr}_{14-\mathrm{x}} \mathrm{Ca}_{\mathrm{x}} \mathrm{Cu}_{24} \mathrm{O}_{41}$. In this work, we calculate the conductivity for doped two-leg ladders using a model of hole-pairs forming a strongly correlated liquid - a single component Luttinger liquid in the presence of disorder. Quantum interference effects are handled using renormalization group methods. We find that our model can account for the low energy features of the experimental results. However, at higher energies the experiments show deviations from the predictions of this model. Using the results of our calculations as well as results on the ground state of doped two-leg ladders, we suggest a scenario to account for the higher energy features of the experimental results.
\end{abstract}

Over the last few years, there has been considerable interest in ladder materials. In particular, the two-leg ladder has received quite a bit of attention. Theoretically, the two-leg ladder is popular becase one can do controlled calculations, both analytically and numerically. Experimentally, two-leg ladders are popular because there are several known two-leg ladder materials, and these materials can be fabricated in a controlled way. In particular, the two-leg ladder material $\mathrm{Sr}_{14-\mathrm{x}} \mathrm{Ca}_{\mathrm{x}} \mathrm{Cv}_{44} \mathrm{O}_{41}$ has received a considerable amount of attention. 2 Unlike some of the other ladder materials which must be fabricated under high pressure, this material can be fabricated under ambient pressure. Also, when Ca replaces some of the $\mathrm{Sr}$, holes are doped into the ladders. However, these holes were already present in the material in the charge reservoir layers composed of $\mathrm{CuO}_{2}$ chains; doping with Ca simply moves them to the ladders. Therefore, this material is self-doped, and hence, extremely clean. Besides these practical reasons, the two-leg ladder is interesting because its properties are remarkably similar to the cuprate superconductors, namely when doped with holes, the system has a spin gap and the doped holes form pairs with a $d_{x^{2}-y^{2}}$-like internal structure.

Recently, conductivity measurements were performed on the hple-doped two-leg ladder material $\mathrm{Sr}_{14-\mathrm{x}} \mathrm{Ca}_{\mathrm{x}} \mathrm{Cu}_{24} \mathrm{O}_{41} \mathrm{~B}^{3}$ The $c$-axis resistivity, $\rho_{c}$, (i.e. the resistivity parallel to the chains of the ladder) is insulating at low temperatures and shows metallic $T$ dependence above $T_{0} \approx 100 \mathrm{~K}$ for $x=11 ; \rho_{c}$ is nonmetallic over the entire region $T<300 K$ for $x=8$. The $a$-axis resistivity, $\rho_{a}$, (i.e. the resistivity parallel to the rungs of the ladder) is insulating at low temperatures and shows a gradual change of sign in the $T$ coefficient at $T^{*} \approx 250 \mathrm{~K}$ for $x=11 ; \rho_{a}$ is insulating for $x=8$. (See Fig. 1 of Ref. 3.)

The $a$-axis optical conductivity, $\sigma_{1 a}(\omega)$, shows pseudogap behavior in the low frequency region, the depressed spectral weight being transferred into the higher energy region. (See Fig. 2 of Ref. 3.) The $c$-axis optical conductivity, $\sigma_{1 c}(\omega)$, shows a low frequency peak. Unlike a Drude peak, the peak in $\sigma_{1 c}(\omega)$ is located at a finite frequency, $\omega_{0}: \omega_{0} \sim 100 \mathrm{~cm}^{-1}$ for $x=8$ and $\omega_{0} \sim 50 \mathrm{~cm}^{-1}$ for $x=11$. (See Fig. 3 of Ref. 过)
In this work, we calculate the conductivity for doped two-leg ladders using a model of hole-pairs forming a strongly correlated liquid. We focus on the $c$-axis conductivity, but we make some remarks about the $a$-axis conductivity. Our model is motivated by several known facts. First, the two-leg ladder (at half-filling and for not too large dopings) is known to have a spin gap. Second, the holes doped into the two-leg ladder are known to form pairs with an internal $d_{x^{2}-y^{2}}$-like structure. Therefore, for our (effective low-energy) model, we consider a liquid of $d_{x^{2}-y^{2}}$ hole pairs in the presence of (weak) disorder. Due to the one-dimensional nature of the ladder, the pairs do not condense. Rather, they form a strongly correlated liquid - a single-component Luttinger liquid.

It should be noted that our model is not purely phenomenological. At weak coupling, controlled calculations using bosonization have been done. 1 . In these calculations, one gets four bosonic modes - two spin modes and two charge modes. These calculations show that, for not too large dopings, both of the spin modes are gapped and one of the charge modes is gapped. Therefore, the low-energy physics is described by single gapless bosonic charge mode. Numerical work 1 shows that there is no phase transition as one goes from weak to strong coupling. Therefore, the low-energy effective theory in terms of a single gapless bosonic charge mode is still valid. However, the parameters of this effective theory cannot be computed as they can in weak coupling; they must be taken as phenomenological parameters from numerics.

The Hamiltonian we consider is

$$
\begin{aligned}
H & =\frac{v}{2} \int d x\left[K \pi^{2}+\frac{1}{K}\left(\partial_{x} \phi\right)^{2}\right] \\
& +\int d x\left[\xi(x) e^{-i \sqrt{4 \pi} \phi}+\xi^{*}(x) e^{i \sqrt{4 \pi} \phi}\right] .
\end{aligned}
$$

This Hamiltonian was first considered in Ref. 5, and in that work they computed the resistivity, $\rho_{c}(T)$. In this work, we extend their calculation of $\rho_{c}(T)$ to lower temperatures, and we also calculate the optical conductivity, $\sigma_{1 c}(\omega)$. In Eq. 1, $\phi$ and $\pi$ are conjugate fields satisfying $[\phi(x), \pi(y)]=i \delta(x-y) . \quad \xi(x)$ and $\xi^{*}(x)$ are Gaussian random variablest satisfying 


$$
\overline{\xi^{*}\left(x_{1}\right) \xi\left(x_{2}\right)}=\Delta_{\mathcal{D}} \delta\left(x_{1}-x_{2}\right),
$$

where the overbar denotes disorder averaging. $K$, the Luttinger liquid parameter, is determined by the interactions. For attractive interactions, $K>1$; for repulsive interactions, $K<1$. ( $K=1$ for a non-interacting system.) Note that the coupling to disorder is via the $4 k_{F}$ charge density wave (CDW). Due to the gaps in the spin modes and one of the charge modes, the $2 k_{F}$ CDW coupling is irrelevant; the most relevant coupling generated is to the $4 k_{F}$ CDW. For later convenience, we define a dimensionless disorder strength, $\mathcal{D} 12$

$$
\mathcal{D}=\frac{1}{n_{i} E_{0}^{2}} \Delta_{\mathcal{D}},
$$

where $n_{i}$ is the density of impurities and $E_{0}$ is a highenergy cut-off. Since our model is a liquid of $d_{x^{2}-y^{2}}$ hole pairs, $E_{0} \sim$ pair binding energy.

We begin with a standard renormalization group (RG) analysis. In an RG treatment we coarse-grain our system, integrating out high energy degrees of freedom, and deriving an effective theory for the low energy degrees of freedom. The RG equations for the parameters can be obtained in a straightforward way using standard techniques $\mathrm{d}$ They are

$$
\begin{aligned}
\frac{d \mathcal{D}}{d l} & =(3-2 K) D, \\
\frac{d K}{d l} & =-D K^{2}, \\
\frac{d v}{d l} & =-D K v .
\end{aligned}
$$

A few words are in order about the RG equations. From the $\mathrm{RG}$ equation for $\mathcal{D}$, we see that there is a localizationdelocalization transition at $K=3 / 2$. However, for the systems we are considering, $K<1$. Hence, $\mathcal{D}$ is strongly relevant and flows to strong coupling; all states will be localized at low temperatures. Also note that $K$ as well as the speed of the excitations, $v$, decrease under the RG.

From the Kubo formula, the $c$-axis conductivity for our model is given by

$$
\sigma_{1 c}(\omega, T)=\frac{1}{\omega} \operatorname{Im}\left\langle\overline{J(x, t) J\left(x^{\prime}, t^{\prime}\right)}\right\rangle(q=0, \omega),
$$

where $J(x, t)$ is the current operator

$$
J(x, t)=\frac{v}{\sqrt{\pi}} \partial_{t} \phi .
$$

Fourier transforming in space and time, we have

$$
\sigma_{1 c}(\omega, T)=-\frac{v^{2} \omega}{\pi} \operatorname{Im}\left\langle\overline{\phi(x, t) \phi\left(x^{\prime}, t^{\prime}\right)}\right\rangle(q=0, \omega) .
$$

From the RG analysis, we saw that the disorder grows strong at low energies and the system localizes. In this work, we will not be concerned with the properties of the localized (insulating) state. More formally, we will be interested in the properties near the Luttinger liquid (free boson) fixed point. Therefore, we can use RG techniques $\mathrm{d}$ en to compute the conductivity. Consider the Green's function

$$
G(\omega, \mathcal{D})=\left\langle\overline{\phi(x, t) \phi\left(x^{\prime}, t^{\prime}\right)}\right\rangle(q=0, \omega) .
$$

Suppose we scale energies $-\omega \rightarrow b \omega\left(b=e^{d l}\right)$. The Green's function transforms as

$$
G(\omega, \mathcal{D})=b^{2} Z G\left(b \omega, b^{3-2 K} \mathcal{D}\right) .
$$

The factor $b^{2}$ comes from dimensional analysis, while the factor $Z$ is due to the "wave function renormalization" which arises in the RG. For simplicity, we ignore the renormalization of $K$ and $v$. Since, by assumption, the disorder is weak and since we are far from the localization-delocalization transition, the renormalization of $K$ and $v$ should be small. Writing $Z=b^{-\gamma}$, where $\gamma=\mathcal{D} K$, and iterating gives

$$
G(\omega, \mathcal{D})=\exp \left[\int_{0}^{l^{*}} d l(2-\gamma(l))\right] G\left(e^{l^{*}} \omega, e^{(3-2 K) l^{*}} \mathcal{D}\right)
$$

We choose $l^{*}$ such that $e^{l^{*}}=\left(E_{0} / E\right)$, where $E=\omega$ or $T$.

Notice that on the right hand side of Eq. 9, $G$ is evaluated at $E_{0}$. At such high energies, quantum interference effects should be negligible. In this regime, the Born approximation gives accurate results for the conductivity. In the Born approximation, the Green's function has the form

$$
G(\omega, \mathcal{D})=\frac{1}{\omega^{2}+i \omega / \tau} .
$$

The functional form of $\tau$ can be deduced from scaling arguments.11 12 In the Born approximation, $1 / \tau \sim n_{i} v \mathcal{D}$. Also, from scaling

$$
\frac{1}{\tau\left(b E, b^{3-2 K} \mathcal{D}\right)}=\frac{b}{\tau(E, \mathcal{D})},
$$

where $E=\omega$ or $T$. Therefore, we deduce that

$$
\frac{1}{\tau(E, \mathcal{D})}=\text { const } \times n_{i} v \mathcal{D}\left(\frac{E_{0}}{E}\right)^{2-2 K} \text {. }
$$

Knowing the conductivity in the Born approximation, we can use Eq. 9 to relate the conductivity at lower energies, where quantum interference effects are important, to the conductivity at higher energies, where quantum interference effects are negligible and the Born approximation is accurate.

Let us first understand what our expressions tell us about the resistivity, $\rho_{c}(T)$. Using Eqs. 10 and 12 with Eq. 9 gives

$$
\rho_{c}(T) \sim\left(\frac{1}{x}\right)^{2-2 K} \exp \left\{\frac{K \mathcal{D}}{3-2 K}\left[(1 / x)^{3-2 K}-1\right]\right\}
$$


where we have introduced $x=T / E_{0}$. Consider the first factor on the right hand side. This mower-law factor can be deduced by scaling arguments.11 The second factor arises from the "wave function renormalization" in the $\mathrm{RG}$ and is due to quantum interference/localization effects.

Now let us consider the optical conductivity, $\sigma_{1 c}(\omega)$ (at $T=0)$. Again, using Eqs. 10 and 12 with Eq. 9 gives

$$
\sigma_{1 c}(\omega) \sim \frac{x^{2-2 K}}{x^{6-4 K}+\delta^{2}} \exp \left\{\frac{-K \mathcal{D}}{3-2 K}\left[(1 / x)^{3-2 K}-1\right]\right\}
$$

where $\delta \sim n_{i} v \mathcal{D} / E_{0}$ and we have introduced $x=\omega / E_{0}$. Consider the first factor on the right hand side. Notice that for $K=1$, we have a peak at $\omega=0-$ a Drude peak. For $K<1$ (i.e. for an interacting system with repulsive interactions), the peak is shifted to finite frequency. The smaller the value of $K$, the higher the frequency at which the peak occurs. Now consider the second factor. This factor arises from the "wave function renormalization" in the RG. This factor is due to quantum interference/localization effects, and it suppresses the low frequency behavior.

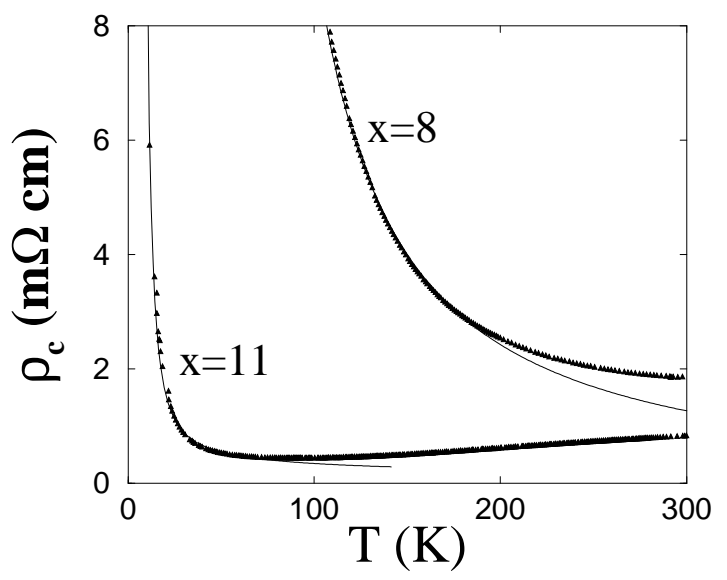

FIG. 1. $\rho(T)$ vs. T. Solid lines are from Eq. 13. The experimental data is from Ref. 3 .

How do our results compare with the experimental results of Ref. 33? In Fig. 1, we plot our calculated result for $\rho_{c}(T)$ as well as the experimental results of Ref. 3. To fit the data for $x=8$, we took $K=.25, \mathcal{D}=0.1$, $E_{0}=425 K$, and $\rho_{0}=0.74 \mathrm{~m} \Omega \mathrm{cm}$. To fit the data for $x=11$, we took $K=0.78, \mathcal{D}=0.1, E_{0}=142 K$, and $\rho_{0}=0.285 \mathrm{~m} \Omega \mathrm{cm}$. For $x=8$, we were able to get a reasonable fit to the data at lower temperatures. However, we cannot account for the rather constant resistivity starting at $\sim 200 K$. We believe that pairing fluctuations and the renormalization of $K$ and $v$, which we ignored in our calculation, could account for this difference. For $x=11$, we were able to get a reasonable fit to the data at low temperatures. However, our calculated result cannot account for the metallic behavior seen above $T_{0} \approx 100 \mathrm{~K}$. We believe the metallic behavior is due to pairing fluctuations, which were not included in our model. (See below for more details on pairing fluctuations.)

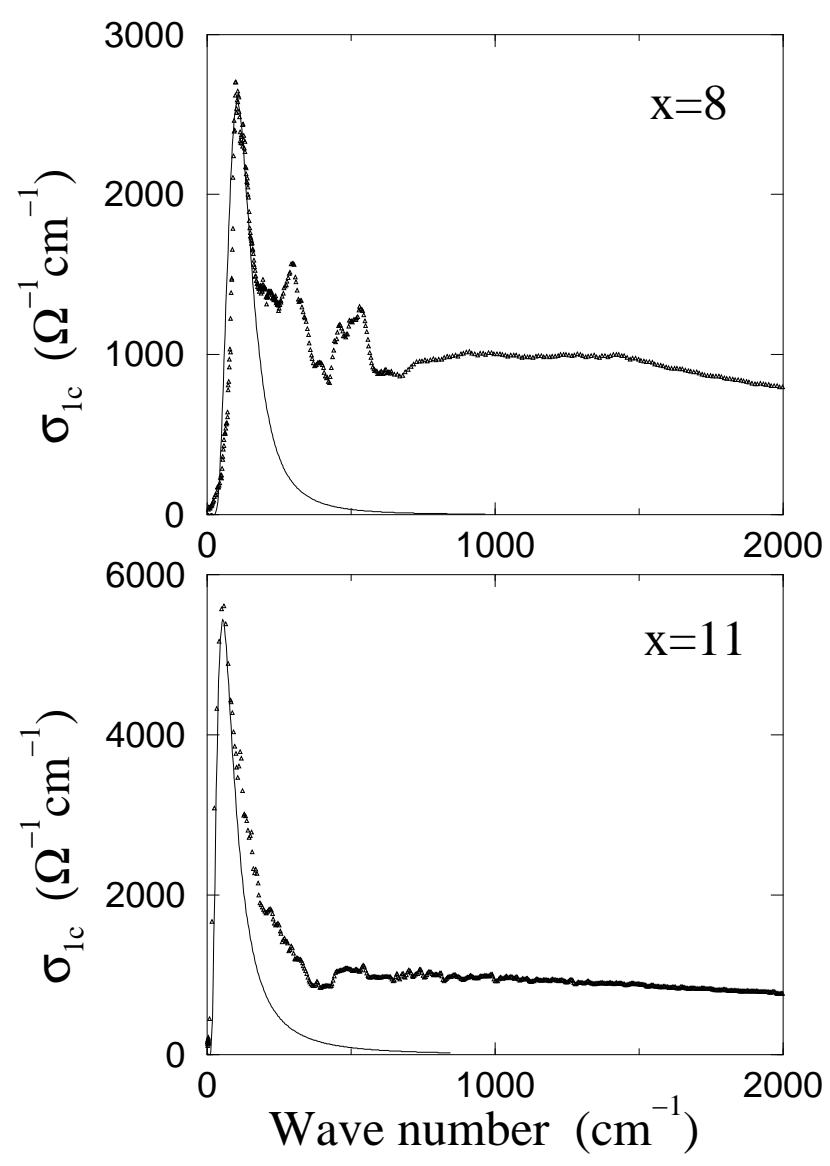

FIG. 2. $\sigma_{1}(\omega)$ vs. Wave number. Solid lines are from Eq. 14. The experimental data is from Ref. 3 at $10 K$.

In Fig. 2 we plot our calculated result for $\sigma_{1 c}(\omega)$ as well as the experimental results of Ref. 3. We see that $\sigma_{1 c}(\omega)$ shows a narrow, finite frequency peak. This finite frequency (rather than $\omega=0$ ) peak is due to the strong correlations; the smaller the value of $K$, the larger the frequency at which the peak occurs. However, at higher energies, our calculated result does not reproduce some of the features of the measured conductivity. Namely, there is appreciable spectral weight at higher energies, while the calculated result gives practically no spectral weight at higher energies. We believe this appreciable spectral weight at higher energies is due to pairing fluctuations, which were not included in our model. In order to fit the data, we took $K=0.25, \mathcal{D}=0.1, \delta=0.075$, $E_{0}=0.04 \mathrm{~cm}^{-1}$, and $\sigma_{0}=150 \Omega^{-1} \mathrm{~cm}^{-1}$ for $\mathrm{x}=8$; we took $K=0.78, \mathcal{D}=0.1, \delta=0.1, E_{0}=0.035 \mathrm{~cm}^{-1}$, and $\sigma_{0}=355 \Omega^{-1} \mathrm{~cm}^{-1}$ for $\mathrm{x}=11$. The values for $E_{0}$ and $\sigma_{0}$ are inconsistent with the values determined from the resistivity. For $x=8$, the peak occurs at $\sim 100 \mathrm{~cm}^{-1}$ $(=144 K)$. At these energies the renormalization of $K$ 
and $v$, which we ignored in our calculation, could be coming into play. For $x=11$, the peak occurs at $\sim 50 \mathrm{~cm}^{-1}$ $(=72 K)$. At these energies, pairing fluctuations are probably still be present and could account for our inability to fit the data consistently. (See below for more details on pairing fluctuations.)

For not too large dopings, a simple picture which captures the essential physics of the ground state of doped two-leg ladders is hole pairs moving through a nearest neighbor resonating valence bond (RVB) background. 13 A typical configuration in the ground state is shown in Fig. 3. In this picture, the spin gap is related to the energy necessary to break the short ranged singlet bonds; pairing also occurs in order to minimize the number of broken singlet bonds. From this picture of the ground state, combined with our calculations for the conductivity, we suggest a scenario to explain the experimental results of Ref. 3 .

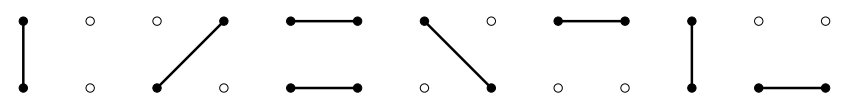

FIG. 3. Typical configuration in the ground state of doped two-leg ladders - hole pairs moving through an RVB background. (Figure taken from Ref. 13.)

$T_{0}(\approx 100 K)$ is the temperature at which the $c$-axis resistivity becomes metallic for $x=11.0$ We believe that this is the temperature at which pairing fluctuations occur. In other words, the RVB background is still, for the most part, in tact. However, the holes are no longer tightly bound into pairs. In the bosonization treatment, pairing is due to the "pinning" of $\theta_{\rho^{-}}=\theta_{\rho, b}-\theta_{\rho, a}$, the relative phase of the charge modes in the bonding and antibonding bands 4 . When $\theta_{\rho^{-}}$is pinned, the relative phase between the bonding and antibonding bands become locked together, with fluctuations being gapped. In our calculation, we took $\theta_{\rho^{-}}$to be pinned; we ignored fluctuations, since they are gapped. We speculate that above $T_{0}, \theta_{\rho^{-}}$is no longer pinned and is free to fluctuate. This also explains why our calculated conductivity has almost no spectral weight at higher energies. Namely, at higher energies the missing spectral weight is due to the fluctuations of $\theta_{\rho^{-}}$which we ignored.

$T^{*}(\approx 250 K)$ is the temperature at which the $a$ axis resistivity becomes metallic for $x=11$; it was found to coincide with the spin-gap inferred from NMR measurements. 3 From the ground state wave function, we see that it is energetically costly for single electrons to tunnel between neighboring ladders as long as the RVB background is in tact i.e. as long as there is a spin-gap. Above $T^{*}$, the spin-gap is destroyed, and hence it is not costly for single electrons to tunnel between neighboring ladders. In the bosonization language, due to the spin gap, single particle tunneling is irrelevant. However, the operator which tunnels a pair between neighboring ladders is generated under the RG. It is the most relevant operator generated, and hence dominates the $a$ axis transport. Once the spin-gap is destroyed, the $a$-axis transport will be dominated by single particle tunneling. $\sigma_{1 a}(\omega)$ was observed to exhibit pseudogap behavior. The pseudogap was identified to open up at $\sim 1100 \mathrm{~cm}^{-1}$ $(=1584 K)$ for $x=8$ and $\sim 600 \mathrm{~cm}^{-1}(=864 K)$ for $x=11$. These values are much larger than the spin-gap values. However, it is possible that the energies at which the pseudogap begins were overestimated in Ref. $3.14 \mathrm{We}$ believe that the pseudogap should coincide with the spingap, namely because that is where single particles become free to tunnel between neighboring ladders.

To summarize, we computed the conductivity of doped two-leg ladders. Our model consisted of hole pairs forming a single component Luttinger liquid in the presence of disorder; quantum interference effects were handled using RG methods. Our results could account for the low-energy features of Ref. 3. However, there were some discrepancies at higher energies, namely we could not account for the metallic behavior in $\rho_{c}(T)$ for $x=11$, and we could not account for the significant spectral weight in $\sigma_{1 c}(\omega)$ at higher energies. Based on the results of our calculations as well as results on the ground state of doped two-leg ladders, we suggested that these discrepancies were due to pairing fluctuations which were not included in our model. We also commented that it is quite natural that $T^{*}$ corresponds to the spin-gap inferred from NMR measurements; single particles are free to tunnel between ladders above $T^{*}$, but not below $T^{*}$. Finally, we commented that we believe the energy at which the pseudogap begins in $\sigma_{1 a}(\omega)$ should coincide with the spin-gap, and that its value may have been overestimated in Ref. 3 .

The author is especially grateful to T. Osafune, N. Motoyama, H. Eisaki, S. Uchida, and S. Tajima for use of their data; to N. Motoyama for correspondence; and to T. Timusk for innumerable helpful discussions about the data. The author would also like to thank A. J. Berlinsky, C. Kallin, D. J. Scalapino, and especially K. M. Kojima and J. Sólyom for helpful discussions and comments about the manuscript. Finally, the author gratefully acknowledges the hospitality of Argonne National Laboratory, where parts of this manuscript were written. This work was supported by the DOE under grants No. 85ER45197 and No. W-31-109-ENG-38.

$\dagger$ present address

${ }^{1}$ For reviews see E. Dagotto and T. M. Rice, Science 271, 618 (1996); E. Dagotto, Rep. Prog. Phys. 62, 1525 (1999).

${ }^{2}$ E. M. McCarron, et. al., Mater. Res. Bull. 23, 1355 (1988).

${ }^{3}$ T. Osafune, et. al., Phys. Rev. Lett. 82, 1313, (1999).

${ }^{4}$ H-H. Lin, L. Balents, and M. P. A. Fisher, Phys. Rev. B. 56, 6569 (1997).

${ }^{5}$ E. Orignac and T. Giamarchi, Phys. Rev. B. 56, 7167 (1997).

${ }^{6}$ R. M. Noack, S. R. White, and D. J. Scalapino, Physica C 270, 281 (1996).

${ }^{7}$ A. A. Abrikosov and J. A. Ryshkin, Adv. Phys., 27, 147 (1978).

${ }^{8}$ See, for example, J. Cardy, Scaling and Renormalization in Statistical Physics, (Cambridge University Press, Cambridge, 1996). 
${ }^{9}$ J. Zinn-Justin, Quantum Field Theory and Critical Phenomena, (Oxford University Press, Oxford, 1993).

${ }^{10}$ D. J. Amit, Field Theory, the Renormalization Group, and Critical Phenomena, (McGraw Hill Inc., Great Britain 1978).

11 T. Giamarchi and H. J. Schulz, Phys. Rev. B 37, 325
(1988).

12 C. L. Kane and M. P. A. Fisher, Phys. Rev. B 56, 15231 (1997).

${ }^{13}$ G. Sierra, M. A. Martin-Delgado, J. Dukelsky, S. R. White, and D. J. Scalapino, Phys. Rev. B 57, 11666 (1998).

${ }^{14} \mathrm{~T}$. Timusk, private communication. 